\title{
Haptoglobin genotype modulates the relationships of glycaemic control with cognitive function in elderly individuals with type 2 diabetes
}

\author{
Elizabeth Guerrero-Berroa • Ramit Ravona-Springer • \\ Anthony Heymann • James Schmeidler • Andrew Levy • \\ Derek Leroith • Michal S. Beeri
}

Received: 12 August 2014 / Accepted: 8 December 2014 / Published online: 28 January 2015

(C) Springer-Verlag Berlin Heidelberg 2015

\begin{abstract}
Aims/hypothesis The purpose of this study was to investigate whether the association of glycaemic control with cognitive function is modulated by the haptoglobin 1-1 (Hp 1-1) genotype in cognitively normal elderly individuals with type 2 diabetes. Methods In this cross-sectional study, we examined 793 participants who were genotyped for Hp (80 Hp 1-1 carriers and 713 Hp 1-1 non-carriers) enrolled in the Israel Diabetes and Cognitive Decline (IDCD) study. Glycaemic control was operationally defined by $\mathrm{HbA}_{1 \mathrm{c}}$ level. The outcome measures
\end{abstract}

E. Guerrero-Berroa $\cdot$ J. Schmeidler $\cdot$ M. S. Beeri

Department of Psychiatry, Icahn School of Medicine at Mount Sinai, New York, NY, USA

E. Guerrero-Berroa $(\bowtie)$

James J. Peters VA Medical Center, 130 West Kingsbridge Road, Room 1F-01, Bronx, NY 10468, USA

e-mail: elizabeth.guerrero-berroa@mssm.edu

R. Ravona-Springer $\cdot$ M. S. Beeri

The Joseph Sagol Neuroscience Center, Sheba Medical Center,

Ramat Gan, Israel

R. Ravona-Springer $\cdot$ A. Heymann

Sackler Faculty of Medicine, University of Tel Aviv, Tel-Aviv, Israel

A. Heymann

Maccabi Healthcare Services, Tel-Aviv, Israel

A. Levy

Technion Faculty of Medicine, Technion Israel Institute of

Technology, Technion, Haifa, Israel

D. Leroith

Department of Medicine, Icahn School of Medicine at Mount Sinai, New York, NY, USA

M. S. Beeri

The School of Psychology, Interdisciplinary Center, Hertzlia, Israel were performance in four cognitive domains (episodic memory, attention/working memory, language/semantic categorisation, executive function) and overall cognition, a composite of the domains. Effect sizes were obtained from hierarchical linear regression analyses for each outcome measure, controlling for demographics, type 2 diabetes-related characteristics, cardiovascular risk factors, and their interactions with Hp genotype.

Results Interaction analyses showed significantly stronger associations of $\mathrm{HbA}_{1 \mathrm{c}}$ with poorer cognitive function among $\mathrm{Hp}$ 1-1 carriers than non-carriers; attention/working memory $(p<0.001)$ and overall cognition $(p=0.003)$. For these two cognitive domains, associations were significant for Hp 1-1 carriers despite the small sample size $(p<0.00001$ and $p=$ 0.001 , respectively), but not for non-carriers.

Conclusions/interpretation Our findings suggest that patients with type 2 diabetes and poor glycaemic control carrying the Hp 1-1 genotype may be at increased risk of cognitive impairment, particularly in the attention/working memory domain. The association of glycaemic control with this domain may indicate cerebrovascular mechanisms.

Keywords Cognition · Cognitive domains · Diabetes · Glucose $\cdot$ Glycaemia $\cdot$ Haptoglobin $\cdot \mathrm{HbA}_{1 \mathrm{c}}$

$\begin{array}{ll}\text { Abbreviations } \\ \text { AD } & \text { Alzheimer's disease } \\ \text { CDR } & \text { Clinical Dementia Rating (scale) } \\ \text { CSF } & \text { Cerebrospinal fluid } \\ \text { GDS } & \text { Geriatric depression scale } \\ \text { Hp } & \text { Haptoglobin } \\ \text { IDCD } & \text { Israel Diabetes and Cognitive Decline (study) } \\ \text { MCI } & \text { Mild cognitive impairment } \\ \text { MHS } & \text { Maccabi Healthcare Services } \\ \text { MMSE } & \text { Mini-mental state examination }\end{array}$


SVD Small vessel disease

WAIS Wechsler Adult Intelligence Scale

WASI Wechsler Abbreviated Scale of Intelligence

\section{Introduction}

Elderly individuals with type 2 diabetes are at increased risk for cognitive compromise and dementia, including vascular dementia and Alzheimer's disease (AD) [1-5], with genetic factors and poor management of type 2 diabetes among possible underlying causes. With the elderly population being the fastest growing segment of the population [6] and the accelerating rates of both type 2 diabetes $[7,8]$ and dementia $[9,10]$, better understanding of known and novel mechanisms that may affect cognition in type 2 diabetes is imperative for enhancement of public health.

The haptoglobin (Hp) gene produces a haemoglobinbinding protein that prevents oxidative tissue damage [11]. A recent study found more downregulation and oxidation of Hp in plasma of patients with mild cognitive impairment and AD compared with age-matched controls [12], and cerebrospinal fluid (CSF) Hp concentration was one of two biomarkers that improved discrimination of $\mathrm{AD}$ vs non-AD dementias, when used in combination with tau levels in comparison with tau alone [13]. Two classes of alleles at the Hp locus (1 and 2) yield three Hp genotypes 1-1, 2-1 and 2-2 [14]. While the Hp 2-2 genotype has been associated with increased type 2 diabetes complications, primarily increased myocardial infarction and mortality risk [15], Hp 1-1 appears to be associated with cerebral small vessel disease (SVD; e.g., lacunar infarcts and white matter lesions), which in turn is associated with deficits in working memory and processing speed [16].

We recently published a study showing that elderly patients with type 2 diabetes carrying the Hp 1-1 genotype have poorer cognitive function than both Hp 1-2 and Hp 2-2 carriers (who have similar cognitive function) [17]. We also noted that the collective association of seven cardiovascular risk factors with cognition was significantly stronger among Hp 1-1 carriers [17]. $\mathrm{HbA}_{1 \mathrm{c}}$, the gold standard indicator of glycaemic control and a consistent predictor of type 2 diabetes micro- and macrovascular complications and of ischaemic stroke [18], has been associated with cognitive dysfunction and decline among cognitively normal elderly individuals with [5] and without type 2 diabetes [19]. Similarly, $\mathrm{HbA}_{1 \mathrm{c}}$ has been associated with incident mild cognitive impairment (MCI) or dementia [20]. The possible involvement of the Hp genotype in the association of glycaemic control with cognition may have clinical implications for more effective treatment for type 2 diabetes since some studies have shown that cognition can be improved with successful glycaemic control $[21,22]$. Thus, this study investigated whether the association of glycaemic control with cognitive function is modulated by the $\mathrm{Hp} 1-1$ genotype in cognitively normal patients with type 2 diabetes. In addition, for descriptive purposes, cognitive functions were stratified into quartiles.

\section{Methods}

Participants This study builds on the Israel Diabetes and Cognitive Decline (IDCD) study, which investigates the effects of long-term type 2 diabetes-related characteristics on cognitive decline. The IDCD study design has been previously described in detail [17]. Briefly, the IDCD recruited community-dwelling elderly individuals with type 2 diabetes ( $65+$ years old) living in central Israel, from approximately 11,000 clients enrolled in the diabetes registry of the Maccabi Healthcare Services (MHS). MHS is the second largest health maintenance organisation (HMO), treating a representative cross-section of 2 million citizens. The MHS diabetes registry was established in 1998 to facilitate diabetes management and to improve treatment. Any of the following criteria are sufficient for enrolment into the registry: (1) $\mathrm{HbA}_{1 \mathrm{c}}>7.25 \%$ (55.7 mmol/mol); (2) glucose $>11.10 \mathrm{mmol} / \mathrm{l}$ on two exams more than 3 months apart; (3) purchase of diabetic medication twice within 3 months supported by an $\mathrm{HbA}_{1 \mathrm{c}}>6.5 \%(47.5 \mathrm{mmol} / \mathrm{mol})$ or glucose $>6.94 \mathrm{mmol} / \mathrm{l}$ within half a year; (4) diagnosis of type 2 diabetes (ICD-9 code [www.icd9data.com/2007/Volume1]) by a general practitioner, internist, endocrinologist, ophthalmologist or type 2 diabetes advisor, supported by an $\mathrm{HbA}_{\mathrm{lc}}>6.5 \%$ (47.5 mmol/ $\mathrm{mol}$ ) or glucose $>6.94 \mathrm{mmol} / \mathrm{l}$ within half a year. These criteria have been validated by 20 physicians in the MHS against their own practice records [23]. IDCD inclusion criteria were having type 2 diabetes; normal cognition at entry; being free of any neurological (e.g., Parkinson's disease, stroke), psychiatric (e.g., schizophrenia) or other diseases (e.g., alcohol or drug abuse) that might affect cognition; and having an informant. Participants were assessed by a physician experienced in assessment and diagnosis of dementia, and by a neuropsychologist, who administered the broad neuropsychological battery.

The electronic medical records of potential participants are screened by the MHS team for diagnosis of dementia and its subtypes, and for cholinesterase inhibitors. Then MHS personnel ask potential participants on the phone, whether a doctor has ever told them that they have a memory problem, or if they have ever been treated for a memory problem. Participants responding positively are excluded from the study. Potential participants who pass this screen are then assessed by a physician at the Sheba Medical Center for dementia, and are administered the Clinical Dementia Rating (CDR) scale [24].

The CDR scale assesses the severity of cognitive and functional impairment in six domains (memory, orientation, judgment and problem solving, community affairs, home and hobbies, and personal care) through an interview with the participant and an informant. A score of 0 represents normal 
cognition (an inclusion criteria for the IDCD study), 0.5 represents questionable dementia and scores of 1 to 3 reflect increasing severity of dementia $[24,25]$.

All participants are discussed by a diagnostic consensus conference that includes neurologists, psychiatrists and neuropsychologists experienced with dementia, with at least two specialties present. It is important to note that the neuropsychological battery is not used in the process of screening for normal cognition since it is used to calculate the cognitive outcome measures. Those with a CDR $>0$ (reflecting questionable dementia or increasing levels of dementia severity) are excluded from the study and referred back to their primary physician. The Mini-Mental State Examination (MMSE) [26], which summarises various areas of cognitive functions (orientation, concentration, memory, language and visual construction), is used to describe the general level of cognitive performance of the sample.

Analyses include prospective historical diabetes-related data from the MHS and the baseline cognitive data collected by the IDCD. Longitudinal IDCD assessments are ongoing.

The sample for this study consisted of 793 IDCD participants with normal cognitive function as described above. All participants had complete data on Hp genotyping, cognitive domains, demographic characteristics, type 2 diabetes-related characteristics and cardiovascular risk factors (for details, see below). Of the 793 participants, 80 were Hp 1-1 carriers and 713 were Hp 1-1 non-carriers. The study was approved by the Icahn School of Medicine at Mount Sinai, Sheba Medical Center, and MHS IRB committees. All participants provided signed informed consent.

Cognitive function/outcomes Cognitive function at entry was assessed using 12 neuropsychological tests, grouped into cognitive domains according to the factor with the highest loading: episodic memory: word list memory, word list recall and word list recognition from the Consortium to Establish a Registry for Alzheimer's Disease (CERAD) neuropsychological battery [27, 28]; attention/working memory: shape cancellation and digit span (forward and backward) from the Wechsler Memory Scale-Revised (WMS-R) [29]; language/ semantic categorisation: similarities [30], letter fluency [31] and animal fluency [32]; and executive function: trail making test (A and B) [33], CERAD-constructional praxis and digit symbol from the Wechsler Adult Intelligence Scale (WAIS)Revised (WAIS-R) [30]. Raw scores were converted to $z$ scores using participants' means and SDs. A composite measure of global cognitive function (overall cognition) was created by averaging all the $z$ scores. Scores for the four cognitive domains were calculated as averages of $z$ scores.

Glycaemic control/Hb $A_{1 c}$ Glycaemic control was operationally defined as the average $\mathrm{HbA}_{1 \mathrm{c}}$ level across all measurements available for a participant at the MHS diabetes registry in an effort to obtain a stable long-term average, as opposed to a less stable single observation. However, to verify the robustness of the results using this definition, we also performed secondary analyses using the first and last $\mathrm{HbA}_{1 \mathrm{c}}$ measurement available in the MHS diabetes registry, representing the farthest and closest $\mathrm{HbA}_{1 \mathrm{c}}$ measurements in relation to the IDCD baseline cognitive assessment. $\mathrm{HbA}_{1 \mathrm{c}}$ was assessed using standard methods: ion exchange, high performance liquid chromatography. Participants were typically assessed under fasting conditions annually at the MHS.

Covariates Three sets of covariates were used for analyses: demographic characteristics (age, years of education and sex), type 2 diabetes-related characteristics (number of follow-up years in the registry, a surrogate for duration of disease [34] and whether medication for type 2 diabetes was taken [no medication, hypoglycaemic medication, and insulin or insulin + hypoglycaemic medication]) and cardiovascular risk factors (BMI, creatinine, total cholesterol, triacylglycerols, and diastolic and systolic BP). The cardiovascular risk factors were calculated as the means of all assessments for each participant in the MHS diabetes registry. Number of follow-up years in the registry-with an average of 10.5 years - was interpreted as a truncated surrogate for duration of type 2 diabetes. Type of medication taken in the diabetes registry was noted. Another covariate was evaluated in supplementary analyses - extent of depressive symptoms (associated with both type 2 diabetes and cognition), as measured by the 15 -item Geriatric Depression Scale (GDS) [35]. With the exception of demographic characteristics and depressive symptoms, measured at baseline of the IDCD, all covariates were retrieved from the MHS diabetes registry. All these covariates are potential confounders that have been associated with cognitive function [17,36], and thus may account for some of the variance in cognition.

Statistical analyses The independent sample $t$ test and Pearson's $\chi^{2}$ test were used to evaluate differences in demographic and clinical characteristics of the participants by $\mathrm{Hp}$ genotype (Table 1). Hierarchical linear regression analyses evaluated the association of $\mathrm{HbA}_{1 \mathrm{c}}$ with each cognitive outcome, controlling for the three sets of covariates (demographic characteristics, type 2 diabetes-related characteristics and cardiovascular risk factors). The effect size was the partial correlation coefficient.

Hierarchical linear regression analyses were performed for each cognitive outcome to evaluate whether the association of glycaemic control with cognition was modified by Hp genotype. Since we have previously shown in the IDCD participants that Hp 1-1 carriers have lower cognitive function than both Hp 1-2 and Hp 2-2 carriers [17], and to minimise adjustment for multiple comparisons, Hp genotype was evaluated as a dichotomy (Hp 1-1 vs non-Hp1-1 carriers). $\mathrm{HbA}_{1 \mathrm{c}}$ mean for a period averaging 10.5 years was the measure of glycaemic 
Table 1 Demographic and clinical characteristics of the participants by Hp genotype

\begin{tabular}{|c|c|c|c|}
\hline & Hp 1-1 & Non-Hp 1-1 & $p$ value \\
\hline$N$ & 80 & 713 & - \\
\hline Age & $73.7 \pm 5.0$ & $72.7 \pm 4.5$ & 0.07 \\
\hline Education & $12.7 \pm 3.7$ & $13.2 \pm 3.4$ & 0.21 \\
\hline Male $(\%)$ & 56.2 & 61.4 & 0.37 \\
\hline $\begin{array}{l}\text { No. of follow-up years } \\
\text { in the registry }\end{array}$ & $10.5 \pm 1.7$ & $10.5 \pm 1.4$ & 0.76 \\
\hline BMI $\left(\mathrm{kg} / \mathrm{m}^{2}\right)$ & $28.3 \pm 4.6$ & $28.4 \pm 4.4$ & 0.88 \\
\hline Creatinine $(\mu \mathrm{mol} / \mathrm{l})$ & $88.4 \pm 17.7$ & $88.4 \pm 26.5$ & 0.19 \\
\hline Total cholesterol (mmol/1) & $4.7 \pm 0.6$ & $4.7 \pm 0.7$ & 0.46 \\
\hline Triacylglycerols (mmol/l) & $1.7 \pm 1.5$ & $1.8 \pm 1.7$ & 0.37 \\
\hline Diastolic BP (mmHg) & $77.5 \pm 5.3$ & $76.7 \pm 4.8$ & 0.21 \\
\hline Systolic BP (mmHg) & $135.9 \pm 9.0$ & $134.8 \pm 9.5$ & 0.31 \\
\hline $\mathrm{HbA}_{1 \mathrm{c}}, \%$ & $6.8(0.9)$ & $6.8(0.8)$ & 0.77 \\
\hline $\mathrm{HbA}_{1 \mathrm{c}}, \mathrm{mmol} / \mathrm{mol}$ & 50.8 & 50.8 & \\
\hline $\mathrm{HbA}_{1 \mathrm{c}}$ variability & $0.6 \pm 0.4$ & $0.6 \pm 0.4$ & 0.86 \\
\hline Type 2 diabetes medication (\%) & & & 0.46 \\
\hline No medication & 15.0 & 12.1 & \\
\hline Hypoglycaemic medication & 72.5 & 78.5 & \\
\hline $\begin{array}{l}\text { Insulin or insulin }+ \\
\text { hypoglycaemic medication }^{\mathrm{a}}\end{array}$ & 12.5 & 9.4 & \\
\hline GDS & $2.0 \pm 2.0$ & $2.2 \pm 2.4$ & 0.57 \\
\hline MMSE score ${ }^{\mathrm{b}}$ & $27.5 \pm 2.0$ & $28.1 \pm 1.8$ & $0.02 *$ \\
\hline
\end{tabular}

Data are presented as means $\pm \mathrm{SD}$, unless otherwise indicated

a 72 participants received hypoglycaemic medication and five received only insulin; therefore, they were combined

${ }^{\mathrm{b}} n=712$ for non-Hp $1-1$

$p$ value by Student's $t$ test, or Pearson's $\chi^{2}$ for percentages

$* p<0.05$ control. The primary analysis entered all three sets of covariates (demographic characteristics, type 2 diabetes-related characteristics and cardiovascular risk factors), Hp genotype, the interactions of Hp genotype with the three sets of covariates, $\mathrm{HbA}_{1 \mathrm{c}}$ and interaction of $\mathrm{Hp}$ genotype with $\mathrm{HbA}_{1 \mathrm{c}}$. Each interaction was calculated as the product of the $\mathrm{Hp}$ genotype and the other variable. The effect size was the partial correlation coefficient. For a two-sided test at the 0.05 level of significance and an effect size of 0.106 - the smallest significant result in Table $2\left(\mathrm{HbA}_{1 \mathrm{c}}\right.$ mean $\times \mathrm{Hp}$ genotype, footnote b) - the statistical power is 0.84 .

For each subsection of Tables 2, 3 and 4, results were considered significant if they met criteria by the Holm multiple comparisons procedure [37], an enhancement of the Bonferroni inequality, for each cognitive outcome.

To clarify the $\mathrm{Hp}$ genotype differences reflected in the primary analyses, hierarchical linear regression analyses were performed for each cognitive domain and overall cognition, separately for each Hp genotype. The same covariates were used as in the primary analysis: demographic characteristics, type 2 diabetes-related characteristics, cardiovascular risk factors, and $\mathrm{HbA}_{1 \mathrm{c}}$. From these analyses, we obtained the partial correlation of $\mathrm{HbA}_{1 \mathrm{c}}$ with cognition controlling for demographics, type 2 diabetes-related characteristics and cardiovascular risk factors. Fisher's $z$ transformation was used to compare the partial correlations of Hp 1-1 carriers and non-carriers in order to describe the modulating effect of the Hp genotype. The results of these analyses are presented in Table 3.

To concretely describe the different contribution of $\mathrm{Hp}$ genotype to the relationship of $\mathrm{HbA}_{1 \mathrm{c}}$ with cognitive function, the distribution of $\mathrm{HbA}_{1 \mathrm{c}}$ mean was stratified into quartiles for each of the three genotypes. Figure 1 presents, for each $\mathrm{Hp}$ genotype, the estimated mean of each cognitive outcome by

Table 2 Associations of $\mathrm{HbA}_{1 \mathrm{c}}$ with cognition and interactions of $\mathrm{HbA}_{1 \mathrm{c}}$ and $\mathrm{Hp}$ genotype on cognition

\begin{tabular}{|c|c|c|c|c|c|c|c|c|c|}
\hline \multirow[t]{2}{*}{ Cognitive domain } & \multicolumn{3}{|c|}{$\mathrm{HbA}_{1 \mathrm{c}}$ and cognition ${ }^{\mathrm{a}}$} & \multicolumn{3}{|c|}{$\mathrm{HbA}_{1 \mathrm{c}}$ mean $\times$ Hp genotype ${ }^{\mathrm{b}}$} & \multicolumn{3}{|c|}{$\mathrm{HbA}_{1 \mathrm{c}}$ mean $\times \mathrm{Hp}$ genotype $\mathrm{c}^{\mathrm{c}}$} \\
\hline & $F(d f=1,780)$ & Effect size & $p$ value & $F(d f=1,767)$ & Effect size & $p$ value & $F(d f=1,783)$ & Effect size & $p$ value \\
\hline Episodic memory & 1.619 & -0.046 & 0.204 & 2.956 & 0.062 & 0.086 & 0.675 & 0.029 & 0.412 \\
\hline $\begin{array}{l}\text { Language/semantic } \\
\text { categorisation }\end{array}$ & 5.164 & -0.081 & $0.023^{*}$ & 2.850 & 0.061 & 0.092 & 3.780 & 0.069 & 0.052 \\
\hline Attention/working memory & 0.932 & -0.035 & 0.335 & 15.105 & 0.139 & $<0.001^{* * *}$ & 8.756 & 0.105 & $0.003 * *$ \\
\hline Executive function & 13.010 & -0.128 & $<0.001 * * *$ & 1.750 & 0.048 & 0.186 & 0.693 & 0.030 & 0.405 \\
\hline Overall cognition & 9.085 & -0.107 & $0.003 * *$ & 8.725 & 0.106 & $0.003 * *$ & 5.012 & 0.080 & $0.025^{*}$ \\
\hline
\end{tabular}

${ }^{\text {a }}$ Controlling for covariates (age, sex, education, number of follow-up years in the registry, type 2 diabetes medication, BMI, creatinine, total cholesterol, triacylglycerols, and diastolic and systolic BP)

${ }^{\mathrm{b}}$ Controlling for covariates (age, sex, education, number of follow-up years in the registry, type 2 diabetes medication, BMI, creatinine, total cholesterol, triacylglycerols, and diastolic and systolic BP), Hp genotype, interactions of covariates with $\mathrm{Hp}$ genotype and $\mathrm{HbA}_{1 \mathrm{c}}$

${ }^{\mathrm{c}}$ Controlling for covariates (age, sex and education), Hp genotype, interactions of covariates with $\mathrm{Hp}$ genotype and $\mathrm{HbA}_{1 \mathrm{c}}$

$* p<0.05$

${ }^{* *} p<0.01$ and significant by Holm multiple comparisons procedure, ${ }^{* * *} p<0.001$ and significant by Holm multiple comparisons procedure 
Table 3 Effect sizes of linear associations of $\mathrm{HbA}_{1 \mathrm{c}}$ with cognitive domains by Hp genotype

\begin{tabular}{|c|c|c|c|c|c|}
\hline \multirow[t]{2}{*}{ Cognitive domain } & \multicolumn{2}{|c|}{ Hp 1-1 $(n=80)$} & \multicolumn{2}{|c|}{ Non-Hp 1-1 $(n=713)$} & \multirow[t]{2}{*}{$p$ of difference } \\
\hline & Partial $r$ & $p$ & Partial $r$ & $p$ & \\
\hline Episodic memory & -0.257 & $0.033^{*}$ & -0.028 & 0.453 & 0.068 \\
\hline Language/semantic categorisation & -0.263 & $0.029^{*}$ & -0.054 & 0.154 & 0.095 \\
\hline Attention/working memory & -0.509 & $<0.001 * * *$ & 0.015 & 0.700 & $<0.001 * * *$ \\
\hline Executive function & -0.238 & $0.049^{*}$ & -0.108 & $0.004^{* *}$ & 0.297 \\
\hline Overall cognition & -0.414 & $0.001 * * *$ & -0.068 & 0.072 & $0.004 * *$ \\
\hline
\end{tabular}

Controlling for age, sex, education, number of follow-up years in the registry, type 2 diabetes medication, BMI, creatinine, total cholesterol, triacylglycerols, and diastolic and systolic BP

$* p<0.05$

${ }^{* *} p<0.01$ and significant by Holm multiple comparisons procedure, ${ }^{* *} p<0.001$ and significant by Holm multiple comparisons procedure

$\mathrm{HbA}_{1 \mathrm{c}}$ quartiles. Estimated means were derived from analyses of covariance controlling for the three sets of covariates.

In light of recent studies suggesting that the variability in $\mathrm{HbA}_{1 \mathrm{c}}$ is associated with some diabetes complications [38, 39] and the availability of $\mathrm{HbA}_{1 \mathrm{c}}$ observations over an extended period, we performed secondary analyses, in which we compared the associations of variability of glycaemic control (using the SD of each participant's $\mathrm{HbA}_{1 \mathrm{c}}$ values, $\mathrm{HbA}_{1 \mathrm{c}}-\mathrm{SD}$ ) with cognition across the Hp genotypes. In view of the strong correlation between mean and SD of $\mathrm{HbA}_{1 \mathrm{c}}(r=0.498$, $p<0.0005)$, the $\mathrm{HbA}_{1 \mathrm{c}}$ mean was used as an additional covariate.

\section{Results}

Table 1 presents the demographic and clinical characteristics of the sample by Hp genotype. For the entire sample $(n=793)$, mean age was $72.8(\mathrm{SD}=4.6)$ and mean education was 13.2

Table 4 Interactions of first and last $\mathrm{HbA}_{1 \mathrm{c}} \times \mathrm{Hp}$ genotype on cognition

\begin{tabular}{llllll}
\hline Cognitive domains & \multicolumn{2}{l}{$\begin{array}{l}\text { First } \mathrm{HbA}_{1 \mathrm{c}} \times \mathrm{Hp} \\
\text { genotype }\end{array}$} & & \multicolumn{2}{l}{$\begin{array}{l}\text { Last } \mathrm{HbA}_{1 \mathrm{c}} \times \mathrm{Hp} \\
\text { genotype }\end{array}$} \\
\cline { 2 - 3 } \cline { 5 - 6 } & Effect size & $p$ value & & Effect size & $p$ value \\
\hline $\begin{array}{l}\text { Episodic memory } \\
\begin{array}{l}\text { Language/semantic } \\
\text { categorisation }\end{array}\end{array}$ & 0.061 & 0.091 & 0.056 & 0.121 \\
$\begin{array}{l}\text { Attention/working memory } \\
\begin{array}{l}\text { Executive function } \\
\text { Overall cognition }\end{array}\end{array}$ & 0.079 & 0.043 & 0.146 & 0.041 & 0.251 \\
\hline
\end{tabular}

Controlling for covariates (age, sex, education, number of follow-up years in the registry, type 2 diabetes medication, BMI, creatinine, total cholesterol, triacylglycerols, and diastolic and systolic $\mathrm{BP}$ ), Hp genotype, interactions of covariates with $\mathrm{Hp}$ genotype and $\mathrm{HbA}_{1 \mathrm{c}}$

${ }^{* *} p<0.01$ and significant by Holm multiple comparisons procedure
$(\mathrm{SD}=3.4)$. There were more men $(60.9 \%)$ than women. The MMSE mean score was $28.0(\mathrm{SD}=1.8)$, which is consistent with normal cognition. The Hp 1-1 carriers and non-carriers did not differ in any of the characteristics except for the MMSE $(p=0.02)$, with lower scores in Hp 1-1 carriers, which is consistent with our previous findings [17]. There were significant associations of $\mathrm{HbA}_{1 \mathrm{c}}$ with cognition for executive function $(p<0.001)$ and overall cognition $(p=0.003)$, which is also consistent with previous findings from this sample [40].

As presented in Table 2, results from the hierarchical linear regression analyses controlling for demographic characteristics, type 2 diabetes-related characteristics, cardiovascular risk factors and their interactions with Hp genotype showed significant interactions of $\mathrm{Hp}$ genotype with $\mathrm{HbA}_{1 \mathrm{c}}$ for attention/ working memory $(p<0.001)$ and overall cognition $(p=$ $0.003)$. Since the Hp 1-1 carriers and non-carriers differed in MMSE mean scores $(p=0.02)$, the MMSE was included as an additional controlled variable in secondary analyses and results remained essentially unchanged.

Similar results were found when the hierarchical linear regression analyses controlled only for the demographic characteristics and their interactions with Hp genotype (Table 2), with associations being generally only slightly attenuated.

Separate partial correlations for each genotype group show effect sizes of associations of $\mathrm{HbA}_{1 \mathrm{c}}$ with cognition, after controlling for the three sets of covariates. As shown in Table 3, for Hp 1-1 carriers, higher $\mathrm{HbA}_{1 \mathrm{c}}$ was significantly associated with poorer cognitive function in all cognitive domains and overall cognition. For non-carriers, higher $\mathrm{HbA}_{1 \mathrm{c}}$ was only associated with poorer executive function. Comparisons of the partial correlations between the two $\mathrm{Hp}$ genotype groups showed that Hp 1-1 carriers had stronger associations of $\mathrm{HbA}_{1 \mathrm{c}}$ with cognition in attention/working memory $(p=<0.00001)$ and overall cognition $(p=0.004$; Table 3).

Figure 1 depicts the mean and SEM for each quartile of $\mathrm{HbA}_{1 \mathrm{c}}$ for each of the three Hp genotypes (Hp 1-1, Hp 2-1 and 

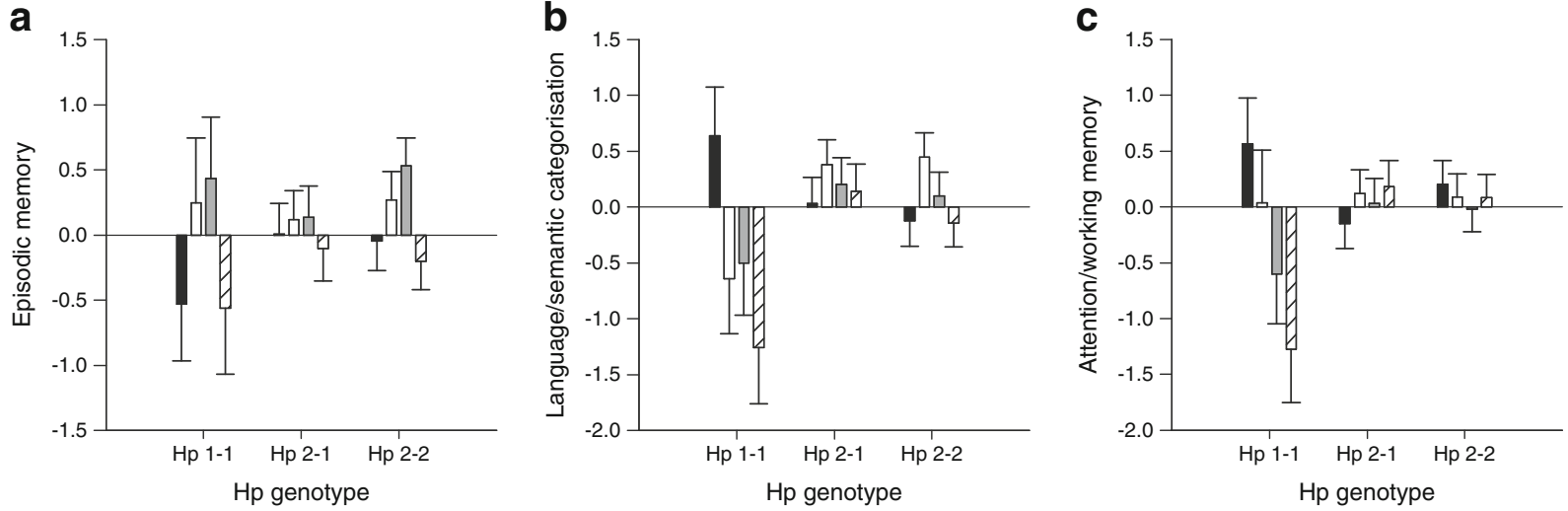

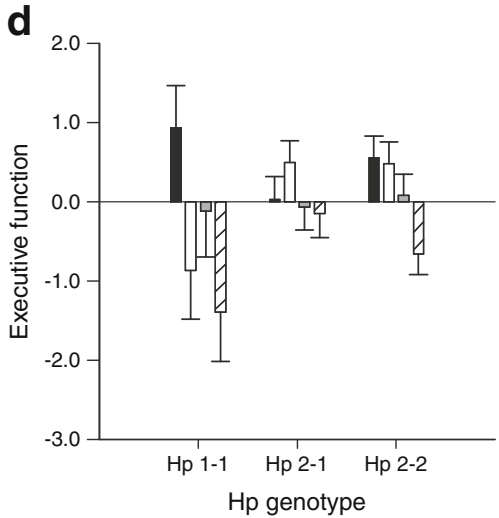

Fig. 1 (a-e) Mean (SEM) of cognitive domains and overall cognition by $\mathrm{Hp}$ genotype and $\mathrm{HbA}_{1 \mathrm{c}}$ mean quartile. Black bar, 1st quartile $\mathrm{HbA}_{1 \mathrm{c}}$ mean; white bar, 2nd quartile $\mathrm{HbA}_{1 \mathrm{c}}$ mean; grey bar, 3rd quartile $\mathrm{HbA}_{1 \mathrm{c}}$ mean; hatched bar, 4th quartile $\mathrm{HbA}_{1 \mathrm{c}}$ mean. Note: controlling for demographic characteristics (age, years of education and sex), type 2 diabetesrelated characteristics (number of follow-up years in the registry, a

Hp 2-2) for each cognitive outcome, controlling for demographic characteristics, type 2 diabetes-related characteristics and cardiovascular risk factors. SEMs for the Hp 1-1 genotype are over twice as big as the other genotypes because the sample size was smaller (Hp 1-1, $n=80$; Hp 2-1, $n=329$; Hp $2-2, n=384)$. Generally, as $\mathrm{HbA}_{1 \mathrm{c}}$ increased, cognitive function decreased. The strength of this association differed by genotype: Hp 1-1 consistently had stronger associations. Additionally controlling for extent of depressive symptoms and its interaction with Hp genotype did not substantially change the results of the primary analyses (Table 2), with the exception of attention/working memory, for which there was a stronger association (effect size $=0.139, p<0.001$ changed to effect size $=0.141, p<0.0001$ ). Results from the secondary analyses comparing the associations of $\mathrm{HbA}_{1 \mathrm{c}}-\mathrm{SD}$ with cognition by Hp genotype showed no significant interaction of $\mathrm{HbA}_{1 \mathrm{c}}$-SD with Hp genotype. Results controlling only for demographic characteristics were essentially identical to those controlling for the larger plethora of covariates.

As shown in Table 4, secondary analyses that used the first and last $\mathrm{HbA}_{1 \mathrm{c}}$ measure showed significant interactions with

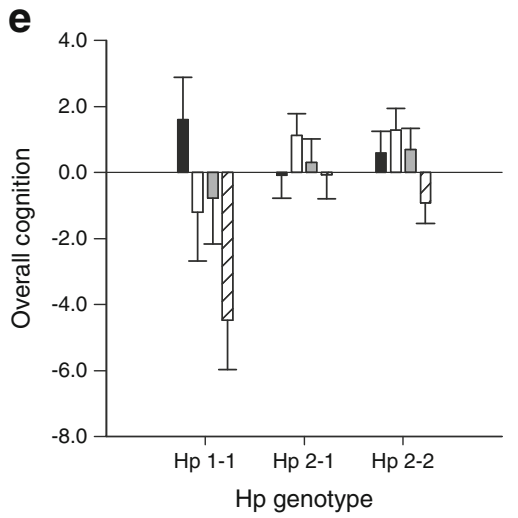

surrogate for duration of disease and whether medication for type 2 diabetes was taken [no medication, hypoglycaemic medication, and insulin or insulin+hypoglycaemic medication]) and cardiovascular risk factors (BMI, creatinine, total cholesterol, triacylglycerols, and diastolic and systolic BP)

Hp genotype for attention/working memory and overall cognition. However, the effect sizes and significance were reduced, reflecting the larger between-subject SD characterising a single observation - rather than an average - due to lower reliability.

IQ is a potential confounder for cognitive performance; we used similarities, one of the four subtests of the Wechsler Abbreviated Scale of Intelligence (WASI), as a proxy for IQ. We repeated the hierarchical linear regression analyses for the $\mathrm{HbA}_{1 \mathrm{c}}$ mean $\times \mathrm{Hp}$ genotype interaction, controlling for similarities. Results remained essentially unchanged (data not shown), including for attention/working memory (effect size $=0.126, p<0.001$ ).

\section{Discussion}

Hp genotype has been shown to be associated with cognitive function [17], and to our knowledge, the present study shows for the first time the differential effect of Hp genotype on the association of glycaemic control with cognition in type 2 
diabetes patients. Our findings show that the Hp genotype modifies the association of glycaemic control with cognition in the domains of attention/working memory and overall cognition, particularly after controlling for demographic characteristics, type 2 diabetes-related characteristics and cardiovascular risk factors. These results were generally less strongly significant when controlling only for demographic variables (age, education and sex), demonstrating the importance of accounting for confounders that may negatively affect cognitive function.

In this sample with type 2 diabetes, one potential biological mechanism that may link the associations among poor glycaemic control, impaired cognition and the Hp genotype is the development of cerebral SVD (e.g., lacunar infarcts and white matter lesions). Relationships between type 2 diabetes and cognitive decline/dementia [3,5], and type 2 diabetes and cerebral SVD [41, 42] have been consistently found. Poor glycaemic control increases the risk of ischaemic stroke [18], associated with cerebral SVD. The latter can affect deleteriously cognitive function, in particular attention/working memory cognitive domains [16, 43, 44], which was affected deleteriously by higher levels of $\mathrm{HbA}_{1 \mathrm{c}}$ in this study.

Hp 1-1 has deleterious effects on endothelial progenitor cells (EPCs), which in turn compromise endothelial repair thus affecting proper functioning of the endothelium. Endothelial dysfunction leads to a deficiency in the forming of new blood vessels and functioning of the blood brain barrier, and is one of the first steps in the progression of cerebral SVD [45, 46]. Hp 1 allele frequency was found to be significantly higher in patients with lacunar stroke than controls [47].

Hp has two polypeptide chains: $\beta$ and $\alpha$. While the $\beta$ chains are identical in all Hp genotypes, Hp 1-1 expresses only $\alpha-1$ chains; Hp 2-1, $\alpha-1$ and $\alpha-2$ chains; and Hp 2-2 only $\alpha-2$ chains [14]. The presence of $\alpha-1 \mathrm{Hp}$ chains has been identified in CSF of patients with $\mathrm{AD}$ and vascular dementia [48], suggesting that Hp 2-1 and Hp 1-1 may be at higher risk of impairment in memory and non-memory cognitive domains. This is consistent with the stronger associations we have found for the overall cognition and non-episodic memory domains in Hp 1-1 carriers.

Elevated concentrations of $\alpha-2 \mathrm{Hp}$ have also been reported in $\mathrm{AD}$ [49] as well as more downregulation and more oxidation of the $\beta \mathrm{Hp}$ chain, which is identical in all Hp genotypes, in plasma of patients with MCI and AD compared with agematched controls [12]. Thus, since studies examining the association of $\mathrm{Hp}$ genotype with cognition are scarce, more studies are warranted to clarify this association as well as the biological mechanisms underlying it.

This study had several limitations including the lack of a non-type 2 diabetes group. Inclusion of such a group would clarify whether the differential effect of Hp genotype on the relationship of glycaemic control with cognition is specifically pertinent to type 2 diabetes or can be generalised. As associations of the Hp genotype with cardiovascular disease have been consistently stronger in type 2 diabetes [50,51], it would not be surprising if associations of $\mathrm{Hp}$ with cognition were also greater in the type 2 diabetes population. The use of follow-up years in the registry as a surrogate for duration of type 2 diabetes was a truncated estimate for all those who already had diabetes when entering the registry, thus the reported values may reflect an under estimate [34]. Another limitation was the use of only one subtest from the WASI, Similarities, as a proxy for IQ. However, one study found that individuals carrying the Hp 2-2 genotype had higher IQ than those with the other genotypes as shown by better performance on a single subtest of the WAIS, Block Design [52]. Also, we controlled for years of formal education, which is highly correlated with IQ [53]. Finally, neuroimaging data was not available and could have helped explore the contribution of brain abnormalities (e.g., cerebral SVD) to possible associations with glycaemic control and to the difference in associations in the Hp subgroups. To the extent that cerebrovascular disease (e.g., cerebral SVD) may be a biological mechanism linking the associations found in this study, excluding participants with stroke (an eligibility criterion of the IDCD study) could have diminished the magnitude of the associations.

Strengths of this study included the availability of information on prospective historical $\mathrm{HbA}_{1 \mathrm{c}}$ permitting an average of 18 measurements - reflecting a relatively stable long-term average rather than a single observation. However, the cognitive outcomes were assessed only once, so the sequence is clear, but causality cannot be established. For example, poorer cognitive performance may affect compliance to medications, negatively affecting glycaemic control. The longitudinal component of the IDCD will shed light on the contributions of Hp genotype and glycaemic control to cognitive decline and dementia. This study included a large sample size, a well characterised diagnosis of type 2 diabetes, well defined and numerous measurements of type 2 diabetes characteristics, and a comprehensive neuropsychological battery. This battery permitted the evaluation of overall cognition and specific cognitive domains, which elucidated attention/working memory as a particularly relevant domain in the context of the relationships of $\mathrm{Hp}$ genotype and glycaemic control. It is important to note that tests assessing the executive function domain, which was unaffected by Hp genotype in the association of glycaemic control with cognition, measure more than one cognitive function or ability. For instance, Trail B measures executive function, mental flexibility and divided attention. Thus, future studies can be aimed at teasing out, in a more granular fashion, specific executive functions that may be affected by Hp genotype.

Our results may have clinical implications since effective regulation of glucose may decrease risk of cognitive impairment and dementia, particularly in type 2 diabetes. Thus, 
future clinical studies should investigate whether glucose lowering or insulin treatments focusing at improving cognition are more beneficial for Hp1-1 carriers than non-carriers. Identifying patients who are most likely to benefit from a treatment is critical for design of clinical trials leading to effectively targeted treatment.

Funding This study was supported by NIA grants R01 AG034087 to MSB and P50 AG005138 to M. Sano, the Helen Bader Foundation and the Irma T. Hirschl Scholar award to MSB, the American Federation for Aging Research (AFAR) Young Investigator award, and the Alzheimer's Association grant NIRG-11-205083 to RRS.

Contribution statement MSB led the development of the study design. RRS and AH collaborated in planning the study design. MSB, RRS, $\mathrm{AH}$ and $\mathrm{AL}$ contributed to the data research. MSB, RRS and $\mathrm{AH}$ contributed to data collection. JS performed the statistical analysis. EGB conducted the literature search and wrote the manuscript. MSB and JS contributed to the writing of the manuscript. All coauthors contributed to the interpretation of results, critically revised the manuscript and gave final approval. EGB is the guarantor of this work.

Duality of interest The authors declare that there is no duality of interest associated with this study.

\section{References}

1. Ahtiluoto S (2010) Diabetes, Alzheimer disease, and vascular dementia: a population-based neuropathologic study. Neurology 75 : $1195-1202$

2. Peila R (2002) Type 2 diabetes, APOE gene, and the risk for dementia and related pathologies: The Honolulu-Asia Aging Study. Diabetes (New York, NY) 51:1256-1262

3. Schnaider Beeri M, Goldbourt U, Silverman JM et al (2004) Diabetes mellitus in midlife and the risk of dementia three decades later. Neurology 63:1902-1907

4. Ravona-Springer R, Schnaider-Beeri M (2011) The association of diabetes and dementia and possible implications for nondiabetic populations. Expert Rev Neurother 11:1609-1617

5. Yaffe K (2012) Diabetes, glucose control, and 9-year cognitive decline among older adults without dementia. Arch Neurol (Chicago) 69:1170-1175

6. US Census Bureau (2012) 2012 National Population Projections: Table 3. Percent distribution of the projected population by selected age groups and sex for the United States: 2015 to 2060 (NP2012-T3). Available from http://www.census.gov/population/projections/data/ national/2012/summarytables.html. Accessed 16 Dec 2013

7. Rathmann W (2004) Global prevalence of diabetes: estimates for the year 2000 and projections for 2030. Diabetes Care 27:2568-2569, author reply 2569

8. Wild S (2004) Global prevalence of diabetes: estimates for the year 2000 and projections for 2030. Diabetes Care 27:1047-1053

9. Gurland BJ, Wilder DE, Lantigua R et al (1999) Rates of dementia in three ethnoracial groups. Int J Geriatr Psychiatr 14:481-493

10. Tang MX, Cross $P$, Andrews $H$ et al (2001) Incidence of $A D$ in African-Americans, Caribbean Hispanics, and Caucasians in northern Manhattan. Neurology 56:49-56

11. Tseng CF (2004) Antioxidant role of human haptoglobin. Proteomics (Weinheim) 4:2221-2228
12. Cocciolo A, Di Domenico F, Coccia R et al (2012) Decreased expression and increased oxidation of plasma haptoglobin in Alzheimer disease: insights from redox proteomics. Free Radic Biol Med 53:1868-1876

13. Abraham J-D (2011) Combined measurement of PEDF, haptoglobin and tau in cerebrospinal fluid improves the diagnostic discrimination between Alzheimer's disease and other dementias. Biomarkers 16: $161-171$

14. Langlois MR, Delanghe JR (1996) Biological and clinical significance of haptoglobin polymorphism in humans. Clin Chem 42:15891600

15. Hochberg I, Roguin A, Nikolsky E, Chanderashekhar PV, Cohen S, Levy AP (2002) Haptoglobin phenotype and coronary artery collaterals in diabetic patients. Atherosclerosis 161:441-446

16. Eilaghi A (2013) Normal-appearing white matter permeability distinguishes poor cognitive performance in processing speed and working memory. Am J Neuroradiol AJNR 34:2119-2124

17. Ravona-Springer R (2013) Haptoglobin 1-1 genotype is associated with poorer cognitive functioning in the elderly with type 2 diabetes. Diabetes Care 36:3139-3145

18. Oh HG (2011) Higher glycated hemoglobin level is associated with increased risk for ischemic stroke in non-diabetic Korean male adults. Diabetes Metab J 35:551-557

19. Ravona-Springer R, Moshier E, Schmeidler J et al (2012) Changes in glycemic control are associated with changes in cognition in nondiabetic elderly. J Alzheimers Dis 30:299-309

20. Yaffe K, Blackwell T, Whitmer RA, Krueger K, Barrett-Connor E (2006) Glycosylated hemoglobin level and development of mild cognitive impairment or dementia in older women. J Nutr Health Aging 10:293-295

21. Meneilly GS (1993) The effect of improved glycemic control on cognitive functions in the elderly patient with diabetes. J Gerontol (Kirkwood) 48:M117-M121

22. Shorr RI (2006) Glycemia and cognitive function in older adults using glucose-lowering drugs. J Nutr Health Aging 10:297-301

23. Heymann AD, Chodick G, Halkin $\mathrm{H}$ et al (2006) The implementation of managed care for diabetes using medical informatics in a large Preferred Provider Organization. Diabetes Res Clin Pract 71:290 298

24. Hughes CP (1982) A new clinical scale for the staging of dementia. Br J Psychiatry 140:566-572

25. Fillenbaum GG (1996) Estimating the validity of the clinical Dementia Rating Scale: the CERAD experience. Consortium to Establish a Registry for Alzheimer's Disease. Aging (Milan, Italy) 8:379-385

26. Folstein MF (1975) "Mini-mental state". A practical method for grading the cognitive state of patients for the clinician. J Psychiatr Res 12:189-198

27. Beeri MS, Schmeidler J, Sano M et al (2006) Age, gender, and education norms on the CERAD neuropsychological battery in the oldest old. Neurology 67:1006-1010

28. Welsh KA, Butters N, Mohs RC et al (1994) The Consortium to Establish a Registry for Alzheimer's Disease (CERAD). Part V. A normative study of the neuropsychological battery. Neurology 44: 609-614

29. Wechsler D (1987) Wechsler Memory Scale — revised manual. Psychological Corporation, San Antonio

30. Godeau P, Frances-Michel C, Wechsler J et al (1981) [Immunofluorescence study of skin biopsies of healthy skin in bacterial endocarditis]. La Revue de medecine interne / fondee par la Societe nationale francaise de medecine interne 2:29-32 [article in French]

31. Spreen O, Benton AL (1977) Neurosensory center comprehensive examination for aphasia (NCCEA), 1977 revision : manual of instructions. Neuropsychology Laboratory, University of Victoria, [Victoria, B.C.] 
32. Newcombe F (1969) Missile wounds of the brain: a study of psychological deficits. Oxford University Press, Oxford

33. Reitan RM (1958) Validity of the Trail Making Test as an indicator of organic brain damage. Percept Mot Skills 8:271-276

34. West RK, Ravona-Springer R, Schmeidler J et al (2014) The association of duration of type 2 diabetes with cognitive performance is modulated by long-term glycemic control. Am J Geriatr Psychiatry 22:1055-1059

35. Sheikh JI, Yesavage JA (1986) Geriatric Depression Scale (GDS): recent evidence and development of a shorter version. Clin Gerontol 5:165-172

36. Wilson RS, Barnes LL, Mendes de Leon CF et al (2002) Depressive symptoms, cognitive decline, and risk of $\mathrm{AD}$ in older persons. Neurology 59:364-370

37. Holm S (1979) A simple sequentially rejective multiple test procedure. Scand J Stat 6:65-70

38. Luk AO (2013) Risk association of HbA1c variability with chronic kidney disease and cardiovascular disease in type 2 diabetes: prospective analysis of the Hong Kong Diabetes Registry. Diabetes Metab Res Rev 29:384-390

39. Penno G, Solini A, Bonora E et al (2013) HbA1c variability as an independent correlate of nephropathy, but not retinopathy, in patients with type 2 diabetes: the renal insufficiency and cardiovascular events (RIACE) Italian Multicenter Study. Diabetes Care 36:2301-2310

40. Ravona-Springer R (2014) Trajectories in glycemic control over time are associated with cognitive performance in elderly subjects with type 2 diabetes. PLoS One 9:e97384

41. Nelson PT, Smith CD, Abner EA et al (2009) Human cerebral neuropathology of type 2 diabetes mellitus. Biochim Biophys Acta (BBA) - Mol Basis Dis 1792:454-469

42. van Harten B (2006) Brain imaging in patients with diabetes: a systematic review. Diabetes Care 29:2539-2548
43. Viana-Baptista M (2008) Cognitive function correlates with frontal white matter apparent diffusion coefficients in patients with leukoaraiosis. J Neurol 255:360-366

44. O'Brien JT (2002) Cognitive associations of subcortical white matter lesions in older people. Ann N Y Acad Sci 977:436-444

45. Rouhl RPW, van Oostenbrugge RJ, Damoiseaux JGMC et al (2009) Haptoglobin phenotype may alter endothelial progenitor cell cluster formation in cerebral small vessel disease. Curr Neurovasc Res 6:3241

46. Rouhl RPW, Mertens AECS, van Oostenbrugge RJ et al (2012) Angiogenic $\mathrm{T}$ cells and putative endothelial progenitor cells in hypertension-related cerebral small vessel disease. Stroke 43:256258

47. Staals J (2008) Haptoglobin polymorphism and lacunar stroke. Curr Neurovasc Res 5:153-158

48. Mattila KM (1994) Altered blood-brain-barrier function in Alzheimer's disease? Acta Neurol Scand 89:192-198

49. Johnson G (1992) Cerebrospinal fluid protein variations in common to Alzheimer's disease and schizophrenia. Appl Theor Electrophor 3: 47-53

50. Levy AP (2004) Haptoglobin: a major susceptibility gene for diabetic cardiovascular disease. Isr Med Assoc J 6:308-310

51. Levy AP (2002) Haptoglobin phenotype is an independent risk factor for cardiovascular disease in individuals with diabetes: The Strong Heart Study. J Am Coll Cardiol 40:1984-1990

52. Mascie-Taylor CG (1985) Associations between some polymorphic markers and variation in IQ and its components in Otmoor villagers. Behav Genet 15:371-383

53. Ritchie SJ (2013) Education is associated with higher later life IQ scores, but not with faster cognitive processing speed. Psychol Aging 28:515-521 